\title{
Toll-Iike receptor genetic variations in bone marrow transplantation
}

\author{
Kaori Uchino ${ }^{1}$, Shohei Mizuno ${ }^{1}$, Aiko Sato-Otsubo' ${ }^{2}$ Yasuhito Nannya ${ }^{2}$, Motonori \\ Mizutani ${ }^{1}$, Tomohiro Horio', Ichiro Hanamura ${ }^{1}$, J. Luis Espinoza ${ }^{3}$, Makoto Onizuka ${ }^{4}$, \\ Koichi Kashiwase ${ }^{5}$, Yasuo Morishima ${ }^{6}$, Takahiro Fukuda7, Yoshihisa Kodera ${ }^{8}$, Noriko \\ Doki $^{9}$, Koichi Miyamura ${ }^{10}$, Takehiko Mori ${ }^{11}$, Seishi Ogawa ${ }^{2}$ and Akiyoshi Takami ${ }^{1}$ \\ ${ }^{1}$ Division of Hematology, Department of Internal Medicine, Aichi Medical University School of Medicine, Nagakute, Japan \\ ${ }^{2}$ Department of Pathology and Tumor Biology, Graduate School of Medicine, Kyoto University, Kyoto, Japan \\ ${ }^{3}$ Cellular Transplantation Biology, Kanazawa University Graduate School of Medical Science, Kanazawa, Japan \\ ${ }^{4}$ Department of Hematology and Oncology, Tokai University School of Medicine, Isehara, Japan \\ ${ }^{5}$ Japanese Red Cross Kanto-Koshinetsu Block Blood Center, Tokyo, Japan \\ ${ }^{6}$ Division of Epidemiology and Prevention, Aichi Cancer Center Research Institute, Nagoya, Japan \\ ${ }^{7}$ Hematopoietic Stem Cell Transplantation Unit, National Cancer Center Hospital, Tokyo, Japan \\ ${ }^{8}$ Department of Promotion for Blood and Marrow Transplantation, Aichi Medical University, Nagakute, Japan \\ ${ }^{9}$ Hematology Division, Tokyo Metropolitan Cancer and Infectious Diseases Center Komagome Hospital, Tokyo, Japan \\ ${ }^{10}$ Department of Hematology, Japanese Red Cross Nagoya First Hospital, Nagoya, Japan \\ ${ }^{11}$ Division of Hematology, Department of Medicine, Keio University School of Medicine, Tokyo, Japan \\ Correspondence to: Akiyoshi Takami, email: takami-knz@umin.ac.jp \\ Keywords: toll-like receptor, unrelated donor, bone marrow transplantation, single nucleotide variation \\ Received: March 15, $2017 \quad$ Accepted: March 31, $2017 \quad$ Published: April 21, 2017 \\ Copyright: Uchino et al. This is an open-access article distributed under the terms of the Creative Commons Attribution License 3.0 \\ (CC BY 3.0), which permits unrestricted use, distribution, and reproduction in any medium, provided the original author and source \\ are credited.
}

\section{ABSTRACT}

The Toll-like receptor family mediates the innate immune system through recognizing the molecular patterns of microorganisms and self-components and leading the synthesis of the inflammatory mediators. We retrospectively examined whether or not genetic variations in toll-like receptor 1 (rs5743551, -7202GQ>A), toll-like receptor 2 (rs7656411, 22215G $>$ T), and toll-like receptor 4 (rs11536889, $+3725 \mathrm{G}>\mathrm{C}$ ) affected transplant outcomes in a cohort of 365 patients who underwent unrelated HLA-matched bone marrow transplantation (for hematologic malignancies through the Japan Marrow Donor Program. Only donor toll-like receptor 4 variation significantly improved the survival outcomes. A multivariate analysis showed that the donor toll-like receptor 4 +3725G/G genotype was significantly associated with a better 5-year progression-free survival and a lower 5-year transplant-related mortality than other variations. Furthermore, the donor toll-like receptor $4+3725 \mathrm{G} / \mathrm{G}$ genotype was associated with a significantly lower incidence of fatal infections than other variations. The validation study of $\mathbf{5 0 2}$ patients confirmed that the donor tolllike receptor $4+3725 \mathrm{G} / \mathrm{G}$ genotype was associated with better survival outcomes. Toll-like receptor4 genotyping in transplant donors may therefore be a useful tool for optimizing donor selection and evaluating pretransplantation risks.

\section{INTRODUCTION}

Allogeneic hematopoietic stem cell transplantation (SCT) is a potentially curative treatment for hematologic malignancies [1]. Despite HLA matching and substantial improvements in supportive care, life-threatening complications, including severe infections, organ damage and graft-versus-host disease (GVHD), remain an enormous obstacle [2]. There is growing evidence that non-HLA genetic variation involved in the immune 
response also represents a significant determinant of outcomes after SCT [3-8].

The toll-like receptor (TLR) family, the most important pattern recognition receptor family, plays a central role in sensing invading pathogens and tissue damage as danger signals, which leads to the massive release of inflammatory mediators such as pro-inflammatory cytokines, reactive oxygen species, antimicrobial peptides and acute-phase proteins into the bloodstream [9, 10]. TLRs are ubiquitously expressed on various cells, such as macrophages, dendritic cells, B cells, T cells, fibroblasts and epithelial cells. Previous reports [11-13] have suggested that TLRs contribute to the inflammatory processes after SCT, where subsequent translocation of bacterial components as well as release of endogenous danger molecules stimulate TLRs to trigger cytokine storm, leading to organ damage, which may vitiate the beneficial anti-microbial effect of TLRs.

Several TLR genes have been investigated in terms of the impact of their variation on outcome and susceptibility to infection and cancer. Among them, 3 functional variations [14-16] in the TLR1 (rs5743551, -7202A $>\mathrm{G})$, TLR2 (rs7656411, 22215G $>\mathrm{T}$ ) and TLR4 $(\mathrm{rs} 11536889,+3725 \mathrm{G}>\mathrm{C})$ genes with high $(>0.2)$ minor allele frequencies in the Asian population were retrospectively investigated to determine their associations with transplant outcomes in a cohort of patients who underwent unrelated HLA-matched bone marrow transplantation (BMT) for hematologic malignancies through the Japan Marrow Donor Program (JMDP).

\section{RESULTS}

\section{Frequencies of TLR genotypes}

The rs5743551 (-7202G $>A)$ variation in the TLR1 gene, the rs7656411 $(22215 \mathrm{G}>\mathrm{T})$ variation in the TLR2 gene and the rs11536889 $(+3725 \mathrm{G}>\mathrm{C})$ variation in the TLR4 gene were genotyped in 365 recipients with hematologic malignancies and their unrelated donors in the discovery cohort (Table 1 ). The frequencies of $\mathrm{A} / \mathrm{A}, \mathrm{A} / \mathrm{G}$ and $\mathrm{G} / \mathrm{G}$ in the TLR1 $-7202 \mathrm{G}>\mathrm{A}$ genetic variant were $13 \%$, $44 \%$ and $44 \%$ in the recipients and $8.8 \%, 41 \%$ and $50 \%$ in the donors $(P=0.52)$, respectively. The frequencies of $\mathrm{G} / \mathrm{G}$, $\mathrm{G} / \mathrm{T}$ and $\mathrm{T} / \mathrm{T}$ in the TLR2 $22215 \mathrm{G}>\mathrm{T}$ genetic variant were $28 \%, 52 \%$ and $19 \%$ in the recipients and $36 \%, 44 \%$ and $20 \%$ in the donors $(P=0.43)$, respectively. The frequencies of $\mathrm{C} / \mathrm{C}, \mathrm{C} / \mathrm{G}$ and $\mathrm{G} / \mathrm{G}$ in the TLR $4+3725 \mathrm{G}>\mathrm{C}$ genetic variant were $7.4 \%, 41 \%$ and $52 \%$ in the recipients and $5.8 \%, 39 \%$ and $55 \%$ in the donors $(P=0.85)$, respectively.

\section{Transplant outcomes according to TLR genotypes}

Univariate analyses (Tables 2 to 4 ) showed that only the donor TLR4 genotype significantly improved the survival outcomes, with the donor $T L R 4+3725 \mathrm{G} / \mathrm{G}$ genotype associated with a better 5-year PFS than the $\mathrm{C} / \mathrm{C}$ or $\mathrm{C} / \mathrm{G}$ genotype $(62 \%$ vs. $43 \%, P=0.0068$; Figure $1 \mathrm{~A})$. Five years was set as the study timepoint according to the median follow-up period among the survivors (970 days; range, 125 to 4798 days). The donor TLR4 $+3725 \mathrm{G} / \mathrm{G}$ genotype also exhibited a trend toward a better 5-year OS (54\% vs. 41\%, $P=0.055$; Figure 1B) and a lower 5-year TRM (23\% vs. 34\%, $P=0.080$; Figure $1 \mathrm{C})$ but did not reduce the 5-year relapse rate $(15 \% \mathrm{vs}$. $23 \%, P=0.12$; Figure 1D). The decrease in the number of analyzed cases regarding the transplant outcomes was due to the lack of data on the survival time and date of relapse in some cases. An analysis by mean imputation for missing data also showed that the donor TLR $4+3725 \mathrm{G} / \mathrm{G}$ genotype was associated with a better 5 -year PFS (52\% vs. 39\%, $P=0.034$ ) and a trend toward a better 5 -year OS (56\% vs. $43 \%, P=0.051)$ compared to other genotypes (Supplementary Table 1), indicating that the presence of the missing data did not markedly impair the results. After adjusting for clinical factors in the multivariate model (Table 5), the donor TLR4 $+3725 \mathrm{G} / \mathrm{G}$ genotype remained associated with a better 5-year PFS (HR, 0.57; 95\% CI, 0.40-0.82; $P=0.0022$ ) and also showed tendencies toward a better 5 -year OS (HR, 0.75; 95\% CI, 0.55-1.0; $P=0.061$ ) and 5-year TRM (HR, 0.63; 95\% CI, 0.40-1.0;P=0.057) than other variations. No other genotypes of TLR1, TLR2 or $T L R 4$ significantly influenced the survival outcomes in multivariate analyses.

When the main causes of TRM were analyzed according to the $T L R 4+3725 \mathrm{G}>\mathrm{C}$ genotype, the donor TLR4 $+3725 \mathrm{G} / \mathrm{G}$ genotype was associated with a significantly lower incidence of fatal infections than other genotypes ( $P=0.047$; Figure 2$)$. The donor TLR4 $+3725 \mathrm{G} / \mathrm{G}$ genotype resulted in one fifth of the cumulative incidence of fatal infection $(0.7 \%$ vs. $4.6 \% ; P=0.11$; Figure $3)$, although there were no statistical differences.

\section{The validation cohort study}

The characteristics of the patients in the validation cohort were similar to those of the patients in the discovery cohort with the exception that the donors in the validation cohort were HLA-DPB1 allele-mismatched. The donor TLR $4+3725 \mathrm{G} / \mathrm{G}$ genotype was associated with a better 5 -year OS (58\% vs. 51\%, $P=0.032$; Figure 4B; Table 6) as well as a trend toward better 5-year PFS (52\% vs. 47\%, $P=0.089$; Figure 4A). According to a multivariate analysis, the donor TLR $4+3725 \mathrm{G} / \mathrm{G}$ genotype remained associated with a significantly better 5-year OS (HR, 0.75; 95\% CI, $0.56-0.99 ; P=0.043$; Table 7$)$ and moderately better 5 -year PFS (HR, 0.81; 95\% CI, 0.62-1.1; $P=0.12$ ).

\section{DISCUSSION}

The discovery cohort study, which consisted of recipient and donor pairs for which the HLA-A, $-B,-C$, 
Table 1: Recipient and donor characteristics in the discovery and validation cohorts

\begin{tabular}{|c|c|c|c|}
\hline Variable & $\begin{array}{l}\text { Discovery cohort } \\
\quad(\mathrm{n}=365)\end{array}$ & $\begin{array}{l}\text { Validation cohort } \\
\qquad(\mathrm{n}=\mathbf{5 0 2})\end{array}$ & $\boldsymbol{P}$ \\
\hline Number of cases & 365 & 502 & \\
\hline Patient age, years, median (range) & $36(1-70)$ & $35(1-67)$ & \\
\hline Donor age, years, median (range) & $33(20-51)$ & & \\
\hline Year of HSCT, median (range) & $2002(1993-2005)$ & $2002(1993-2005)$ & \\
\hline \multicolumn{4}{|l|}{ Patient TLR1 genotype, $\mathrm{n}(\%)$} \\
\hline $\mathrm{A} / \mathrm{A}$ & $46(13)$ & & \\
\hline $\mathrm{A} / \mathrm{G}$ & $160(44)$ & & \\
\hline $\mathrm{G} / \mathrm{G}$ & $159(44)$ & & \\
\hline \multicolumn{4}{|l|}{ Donor TLR1 genotype, n (\%) } \\
\hline $\mathrm{A} / \mathrm{A}$ & $32(8.8)$ & & \\
\hline $\mathrm{A} / \mathrm{G}$ & $150(41)$ & & \\
\hline $\mathrm{G} / \mathrm{G}$ & $183(50)$ & & \\
\hline \multicolumn{4}{|l|}{ Patient TLR2 genotype, $\mathrm{n}(\%)$} \\
\hline $\mathrm{G} / \mathrm{G}$ & $104(28)$ & & \\
\hline $\mathrm{G} / \mathrm{T}$ & $191(52)$ & & \\
\hline $\mathrm{T} / \mathrm{T}$ & $70(19)$ & & \\
\hline \multicolumn{4}{|l|}{ Donor TLR2 genotype, n (\%) } \\
\hline $\mathrm{G} / \mathrm{G}$ & $132(36)$ & & \\
\hline $\mathrm{G} / \mathrm{T}$ & $161(44)$ & & \\
\hline $\mathrm{T} / \mathrm{T}$ & $72(20)$ & & \\
\hline Patient TLR4 genotype, $\mathrm{n}(\%)$ & & & 0.26 \\
\hline $\mathrm{C} / \mathrm{C}$ & $27(7.4)$ & $15(3.0)$ & \\
\hline $\mathrm{C} / \mathrm{G}$ & $149(41)$ & $143(28)$ & \\
\hline $\mathrm{G} / \mathrm{G}$ & $189(52)$ & $344(69)$ & \\
\hline Donor TLR4 genotype, $\mathrm{n}(\%)$ & & & 0.24 \\
\hline $\mathrm{C} / \mathrm{C}$ & $21(5.8)$ & $28(5.6)$ & \\
\hline $\mathrm{C} / \mathrm{G}$ & $142(39)$ & $135(27)$ & \\
\hline $\mathrm{G} / \mathrm{G}$ & $202(55)$ & $339(68)$ & \\
\hline Patient sex, n (\%) & & & 0.65 \\
\hline Male & $225(62)$ & $317(63)$ & \\
\hline Female & $140(38)$ & $185(37)$ & \\
\hline Donor sex, n (\%) & & & 0.53 \\
\hline Male & $241(66)$ & $321(64)$ & \\
\hline Female & $124(34)$ & $181(36)$ & \\
\hline
\end{tabular}

(Continued) 


\begin{tabular}{|c|c|c|c|}
\hline Variable & $\begin{array}{l}\text { Discovery cohort } \\
(n=365)\end{array}$ & $\begin{array}{l}\text { Validation cohort } \\
\qquad(\mathrm{n}=502)\end{array}$ & $\boldsymbol{P}$ \\
\hline Patient/Donor sex match, $\mathrm{n}(\%)$ & & & 0.43 \\
\hline Sex-matched & $253(69)$ & $334(67)$ & \\
\hline Female/Male & $64(18)$ & $86(17)$ & \\
\hline Male/Female & $48(13)$ & $82(16)$ & \\
\hline Disease, $\mathrm{n}(\%)$ & & & 0.0021 \\
\hline AML & $125(34)$ & $147(29)$ & \\
\hline ALL & $82(22)$ & $154(31)$ & \\
\hline MDS & $57(16)$ & $58(12)$ & \\
\hline ML & $36(9.9)$ & $61(12)$ & \\
\hline CML & $65(18)$ & $82(16)$ & \\
\hline Myeloid malignancies & $247(68)$ & $287(57)$ & \\
\hline Lymphoid malignancies & $118(32)$ & $215(43)$ & \\
\hline \multicolumn{4}{|l|}{ Disease stage, $\mathrm{n}(\%)$} \\
\hline High risk & $135(37)$ & & \\
\hline Standard risk & $230(63)$ & & \\
\hline \multicolumn{4}{|l|}{ ABO matching, $\mathrm{n}(\%)$} \\
\hline ABO-matched & $238(65)$ & & \\
\hline Major mismatch & $61(17)$ & & \\
\hline Minor mismatch & $57(16)$ & & \\
\hline Bidirectional & $6(1.6)$ & & \\
\hline Conditioning regimen, $\mathrm{n}(\%)$ & & & 0.015 \\
\hline Myeloablative & $322(88)$ & $467(93)$ & \\
\hline Reduced intensity & $43(12)$ & $35(6.9)$ & \\
\hline \multicolumn{4}{|l|}{$\begin{array}{l}\text { Pretransplantation CMV } \\
\text { serostatus, } \mathrm{n}(\%)\end{array}$} \\
\hline CMV-positive recipient & $224(61)$ & & \\
\hline Missing & $90(25)$ & & \\
\hline $\mathrm{TNC}, \times 10^{8} / \mathrm{kg}$, median (range) & $3.0(0.08-12.3)$ & & \\
\hline Causes of fatal infections, $\mathrm{n}$ & 10 & & \\
\hline Pneumonia, unidentifiable & 4 & & \\
\hline Pneumonia, cytomegalovirus & 2 & & \\
\hline Pneumocystis pneumonia & 1 & & \\
\hline Brain abscess, fungal & 1 & & \\
\hline Sepsis, bacterial & 2 & & \\
\hline
\end{tabular}

TNC, total number of nucleated cells harvested. 
Table 2: The results of a univariate analysis regarding the association between $T L R 1$ variations and clinical outcomes after transplantation in the discovery cohort

\begin{tabular}{|c|c|c|c|c|c|c|c|c|c|}
\hline Variable & $\mathbf{n}$ & $\begin{array}{l}\text { 5-year } \\
\text { OS }\end{array}$ & $P$ & $\begin{array}{l}\text { 5-year } \\
\text { PFS }\end{array}$ & $P$ & 5-year TRM & $P$ & 5-year Relapse & $\boldsymbol{P}$ \\
\hline \multicolumn{10}{|c|}{ Recipient $T L R 1$ genotype } \\
\hline $\mathbf{A} / \mathbf{A}$ & 46 & $47 \%$ & & $57 \%$ & & $26 \%$ & & $17 \%$ & \\
\hline $\mathbf{A} / \mathbf{G}$ & 160 & $47 \%$ & 1.0 & $53 \%$ & 1.0 & $25 \%$ & 1.0 & $22 \%$ & 1.0 \\
\hline G/G & 159 & $49 \%$ & 1.0 & $52 \%$ & 1.0 & $32 \%$ & 1.0 & $16 \%$ & 1.0 \\
\hline G/G & 159 & $49 \%$ & & $52 \%$ & & $32 \%$ & & $16 \%$ & \\
\hline $\mathbf{A} / \mathbf{G}$ or $\mathbf{A} / \mathbf{A}$ & 206 & $47 \%$ & 0.79 & $54 \%$ & 0.94 & $26 \%$ & 0.41 & $20 \%$ & 0.35 \\
\hline \multicolumn{10}{|c|}{ Donor $T L R 1$ genotype } \\
\hline $\mathbf{A} / \mathbf{A}$ & 32 & $54 \%$ & & $62 \%$ & & $29 \%$ & & $8.7 \%$ & \\
\hline $\mathbf{A} / \mathbf{G}$ & 150 & $47 \%$ & 0.92 & $51 \%$ & 0.67 & $28 \%$ & 1.0 & $21 \%$ & 0.70 \\
\hline G/G & 183 & $47 \%$ & 0.92 & $53 \%$ & 0.67 & $28 \%$ & 1.0 & $19 \%$ & 0.70 \\
\hline G/G & 183 & $47 \%$ & & $53 \%$ & & $28 \%$ & & $19 \%$ & \\
\hline $\mathbf{A} / \mathbf{G}$ or $\mathbf{A} / \mathbf{A}$ & 182 & $48 \%$ & 0.81 & $53 \%$ & 0.67 & $28 \%$ & 0.83 & $19 \%$ & 0.76 \\
\hline Variable & n & Grad & $\begin{array}{l}\text { II-IV } \\
\text { GVHD }\end{array}$ & cute & $\boldsymbol{P}$ & $\begin{array}{c}\text { Grade III-IV acute } \\
\text { GVHD }\end{array}$ & $\boldsymbol{P}$ & Chronic GVHD & $P$ \\
\hline \multicolumn{10}{|c|}{ Recipient $T L R 1$ genotype } \\
\hline $\mathbf{A} / \mathbf{A}$ & 46 & & $34 \%$ & & & $9.1 \%$ & & $51 \%$ & \\
\hline $\mathbf{A} / \mathbf{G}$ & 160 & & $31 \%$ & & 1.0 & $11 \%$ & 0.84 & $46 \%$ & 1.0 \\
\hline G/G & 159 & & $38 \%$ & & 1.0 & $15 \%$ & 0.84 & $46 \%$ & 1.0 \\
\hline G/G & 159 & & $38 \%$ & & & $15 \%$ & & $46 \%$ & \\
\hline $\mathbf{A} / \mathbf{G}$ or $\mathbf{A} / \mathbf{A}$ & 206 & & $31 \%$ & & 0.25 & $11 \%$ & 0.20 & $47 \%$ & 0.98 \\
\hline \multicolumn{10}{|c|}{ Donor TLR1 genotype } \\
\hline $\mathbf{A} / \mathbf{A}$ & 32 & & $18 \%$ & & & $0.0 \%$ & & $56 \%$ & \\
\hline $\mathbf{A} / \mathbf{G}$ & 150 & & $39 \%$ & & 0.14 & $16 \%$ & 0.080 & $50 \%$ & 0.59 \\
\hline $\mathbf{G} / \mathbf{G}$ & 183 & & $32 \%$ & & 0.29 & $12 \%$ & 0.10 & $41 \%$ & 0.41 \\
\hline G/G & 183 & & $32 \%$ & & & $12 \%$ & & $41 \%$ & \\
\hline $\mathbf{A} / \mathbf{G}$ or $\mathbf{A} / \mathbf{A}$ & 182 & & $36 \%$ & & 0.53 & $13 \%$ & 0.85 & $51 \%$ & 0.085 \\
\hline
\end{tabular}

-DRB1, -DQB1, and -DPB1 alleles were completely matched, revealed that the donor $\mathrm{G} / \mathrm{G}$ genotype at rs11536889 $(+3725 \mathrm{G}>\mathrm{C})$ of the TLR4 gene predicts significantly better 5-year PFS than other genotypes in patients with hematological malignancies receiving unrelated BMT. The beneficial effects of the donor TLR $4+3725 \mathrm{G} / \mathrm{G}$ genotype were seen on TRM and death attributable to infections but were not evident with respect to GVHD, suggesting that the donor TLR $4+3725 \mathrm{G} / \mathrm{G}$ genotype helps prevent fatal infections. The validation cohort study, which consisted of recipient and donor pairs with one or two HLA-DPB1 mismatched alleles, showed consistent results with the donor TLR $4+3725 \mathrm{G} / \mathrm{G}$ genotype associated with better survival outcomes.

The mechanisms through which the donor TLR4 $+3725 \mathrm{G} / \mathrm{G}$ genotype exerts its beneficial effects remain to be determined. A recent report [16] demonstrated that the TLR4 $+3725 \mathrm{G}>\mathrm{C}$ variation located in its 3 '-untranslated region was functional, and monocytes from $T L R 4+3725 \mathrm{G} / \mathrm{G}$ subjects expressed lower levels 
Table 3: The results of a univariate analysis regarding the association between $T L R 2$ variations and clinical outcomes after transplantation in the discovery cohort

\begin{tabular}{|c|c|c|c|c|c|c|c|c|c|}
\hline Variable & $\mathbf{n}$ & $\begin{array}{l}\text { 5-year } \\
\text { OS }\end{array}$ & $P$ & $\begin{array}{c}\text { 5-year } \\
\text { PFS }\end{array}$ & $P$ & 5-year TRM & $P$ & 5-year Relapse & $P$ \\
\hline \multicolumn{10}{|c|}{ Recipient $T L R 2$ genotype } \\
\hline G/G & 104 & $52 \%$ & & $58 \%$ & & $27 \%$ & & $16 \%$ & \\
\hline G/T & 191 & $47 \%$ & 1.0 & $53 \%$ & 1.0 & $30 \%$ & 1.0 & $17 \%$ & 0.96 \\
\hline $\mathbf{T} / \mathbf{T}$ & 70 & $46 \%$ & 1.0 & $46 \%$ & 1.0 & $25 \%$ & 1.0 & $29 \%$ & 0.62 \\
\hline G/G & 104 & $52 \%$ & & $58 \%$ & & $27 \%$ & & $16 \%$ & \\
\hline $\mathrm{G} / \mathrm{T}$ or $\mathrm{T} / \mathrm{T}$ & 261 & $46 \%$ & 0.93 & $51 \%$ & 0.89 & $29 \%$ & 0.82 & $20 \%$ & 0.64 \\
\hline \multicolumn{10}{|c|}{ Donor $T L R 2$ genotype } \\
\hline $\mathbf{G} / \mathbf{G}$ & 132 & $46 \%$ & & $53 \%$ & & $29 \%$ & & $19 \%$ & \\
\hline G/T & 161 & $52 \%$ & 1.0 & $56 \%$ & 1.0 & $27 \%$ & 1.0 & $17 \%$ & 1.0 \\
\hline $\mathbf{T} / \mathbf{T}$ & 72 & $42 \%$ & 1.0 & $45 \%$ & 1.0 & $31 \%$ & 1.0 & $24 \%$ & 1.0 \\
\hline G/G & 132 & $46 \%$ & & $53 \%$ & & $29 \%$ & & $19 \%$ & \\
\hline $\mathrm{G} / \mathrm{T}$ or $\mathrm{T} / \mathrm{T}$ & 233 & $49 \%$ & 0.91 & $53 \%$ & 0.77 & $28 \%$ & 0.90 & $19 \%$ & 0.74 \\
\hline Variable & n & Grad & $\begin{array}{l}\text { e II-IV } \\
\text { GVHD }\end{array}$ & cute & $P$ & $\begin{array}{c}\text { Grade III-IV acute } \\
\text { GVHD }\end{array}$ & $P$ & Chronic GVHD & $P$ \\
\hline \multicolumn{10}{|c|}{ Recipient $T L R 2$ genotype } \\
\hline G/G & 104 & & $27 \%$ & & & $8.2 \%$ & & $44 \%$ & \\
\hline G/T & 191 & & $39 \%$ & & 0.13 & $14 \%$ & 0.37 & $50 \%$ & 0.47 \\
\hline $\mathbf{T} / \mathbf{T}$ & 70 & & $32 \%$ & & 0.67 & $17 \%$ & 0.29 & $39 \%$ & 0.65 \\
\hline G/G & 104 & & $27 \%$ & & & $8.2 \%$ & & $44 \%$ & \\
\hline $\mathrm{G} / \mathrm{T}$ or $\mathrm{T} / \mathrm{T}$ & 261 & & $37 \%$ & & 0.070 & $14 \%$ & 0.12 & $47 \%$ & 0.42 \\
\hline \multicolumn{10}{|c|}{ Donor $T L R 2$ genotype } \\
\hline G/G & 132 & & $25 \%$ & & & $10 \%$ & & $47 \%$ & \\
\hline G/T & 161 & & $39 \%$ & & 0.054 & $13 \%$ & 1.0 & $51 \%$ & 0.54 \\
\hline $\mathbf{T} / \mathbf{T}$ & 72 & & $39 \%$ & & 0.166 & $16 \%$ & 0.88 & $36 \%$ & 0.48 \\
\hline G/G & 132 & & $25 \%$ & & & $10 \%$ & & $47 \%$ & \\
\hline G/T or $T / T$ & 233 & & $39 \%$ & & 0.015 & $14 \%$ & 0.36 & $46 \%$ & 0.97 \\
\hline
\end{tabular}

Underlined and bold results represent $\mathrm{P}<0.05$.

of TLR4 on their surfaces and were less responsive to lipopolysaccharide (LPS), a TLR4 ligand, than those from $\mathrm{C} / \mathrm{G}$ or $\mathrm{C} / \mathrm{C}$ subjects, possibly due to the preference of microRNAs for binding to the $+3725 \mathrm{G}$ allele over the $+3725 \mathrm{C}$ allele. The lower translational activity associated with the TLR4 $+3725 \mathrm{G} / \mathrm{G}$ genotype may contribute to prevent infection-associated death following SCT.

Evidence of TLR4 activation having a negative influence on outcomes following severe infection has been demonstrated in previous studies using mouse models
[17-19], showing that blockade of TLR4 inhibited systemic inflammatory responses in sepsis, which reduced the disease severity and lethality. Although one may deduce from these findings that the putatively hypoactive TLR 4 $+3725 \mathrm{G} / \mathrm{G}$ genotype increases susceptibility to infectious diseases, the $T L R+3725 \mathrm{G} / \mathrm{G}$ genotype was associated with a lower risk of developing sepsis in China [20], suggesting this to be unlikely. This hypothesis may also be supported by the association of the $T L R+3725 \mathrm{G} / \mathrm{G}$ genotype with a lower rate of Gram-negative infection in sepsis in 
Table 4: The results of a univariate analysis regarding the association between $T L R 4$ variations and clinical outcomes after transplantation in the discovery cohort

\begin{tabular}{|c|c|c|c|c|c|c|c|c|c|}
\hline Variable & $\mathbf{n}$ & $\begin{array}{c}\text { 5-year } \\
\text { OS }\end{array}$ & $P$ & $\begin{array}{l}\text { 5-year } \\
\text { PFS }\end{array}$ & $P$ & 5-year TRM & $P$ & 5-year Relapse & $P$ \\
\hline \multicolumn{10}{|c|}{$\begin{array}{l}\text { Recipient } T L R 4 \\
\text { genotype }\end{array}$} \\
\hline $\mathbf{G} / \mathbf{G}$ & 189 & $48 \%$ & & $52 \%$ & & $30 \%$ & & $18 \%$ & \\
\hline $\mathrm{C} / \mathrm{G}$ & 149 & $50 \%$ & 0.66 & $57 \%$ & 0.49 & $25 \%$ & 0.83 & $18 \%$ & 1.0 \\
\hline $\mathrm{C} / \mathrm{C}$ & 27 & $39 \%$ & 0.33 & $43 \%$ & 0.49 & $33 \%$ & 0.83 & $24 \%$ & 1.0 \\
\hline $\mathbf{G} / \mathbf{G}$ & 189 & $48 \%$ & & $52 \%$ & & $30 \%$ & & $18 \%$ & \\
\hline $\mathrm{C} / \mathrm{G}$ or $\mathrm{C} / \mathrm{C}$ & 176 & $48 \%$ & 0.98 & $54 \%$ & 0.68 & $26 \%$ & 38 & $19 \%$ & 0.65 \\
\hline \multicolumn{10}{|l|}{$\begin{array}{l}\text { Donor } T L R 4 \\
\text { genotype }\end{array}$} \\
\hline $\mathbf{G} / \mathbf{G}$ & 202 & $54 \%$ & & $62 \%$ & & $23 \%$ & & $15 \%$ & \\
\hline $\mathrm{C} / \mathrm{G}$ & 142 & $41 \%$ & 0.23 & $43 \%$ & 0.035 & $35 \%$ & 0.25 & $23 \%$ & 0.50 \\
\hline $\mathrm{C} / \mathrm{C}$ & 21 & $42 \%$ & 0.72 & $44 \%$ & 0.17 & $28 \%$ & 0.85 & $29 \%$ & 0.50 \\
\hline $\mathbf{G} / \mathbf{G}$ & 202 & $54 \%$ & & $62 \%$ & & $23 \%$ & & $15 \%$ & \\
\hline $\mathrm{C} / \mathrm{G}$ or $\mathrm{C} / \mathrm{C}$ & 163 & $41 \%$ & 0.055 & $43 \%$ & 0.0068 & $34 \%$ & 0.080 & $23 \%$ & 0.12 \\
\hline
\end{tabular}

Underlined and bold results represent $P<0.05$.

\begin{tabular}{|c|c|c|c|c|c|c|c|}
\hline Variable & n & $\begin{array}{c}\text { Grade II-IV acute } \\
\text { GVHD }\end{array}$ & $P$ & $\begin{array}{c}\text { Grade III-IV acute } \\
\text { GVHD }\end{array}$ & $P$ & Chronic GVHD & $P$ \\
\hline \multicolumn{8}{|l|}{$\begin{array}{l}\text { Recipient } T L R 4 \\
\text { genotype }\end{array}$} \\
\hline G/G & 189 & $32 \%$ & & $14 \%$ & & $49 \%$ & \\
\hline $\mathrm{C} / \mathrm{G}$ & 149 & $38 \%$ & 0.60 & $12 \%$ & 1.0 & $47 \%$ & 1.0 \\
\hline $\mathrm{C} / \mathrm{C}$ & 27 & $26 \%$ & 0.60 & $7.4 \%$ & 1.0 & $24 \%$ & 0.14 \\
\hline G/G & 189 & $32 \%$ & & $14 \%$ & & $49 \%$ & \\
\hline $\mathrm{C} / \mathrm{G}$ or $\mathrm{C} / \mathrm{C}$ & 176 & $36 \%$ & 0.34 & $12 \%$ & 0.58 & $43 \%$ & 0.53 \\
\hline \multicolumn{8}{|l|}{$\begin{array}{l}\text { Donor TLR4 } \\
\text { genotype }\end{array}$} \\
\hline G/G & 202 & $32 \%$ & & $11 \%$ & & $44 \%$ & \\
\hline $\mathbf{C} / \mathbf{G}$ & 142 & $38 \%$ & 0.68 & $15 \%$ & 0.63 & $49 \%$ & 1.0 \\
\hline $\mathrm{C} / \mathrm{C}$ & 21 & $33 \%$ & 1.0 & $19 \%$ & 0.63 & $53 \%$ & 1.0 \\
\hline G/G & 202 & $32 \%$ & & $11 \%$ & & $44 \%$ & \\
\hline $\mathrm{C} / \mathrm{G}$ or $\mathrm{C} / \mathrm{C}$ & 163 & $37 \%$ & 0.24 & $15 \%$ & 0.18 & $50 \%$ & 0.47 \\
\hline
\end{tabular}

Germany [21], and with a lower susceptibility to severe gastric atrophy related to Helicobacter pylori infection $[22,23]$, hepatitis B recurrence after liver transplantation [24] and advanced periodontitis $[25,26]$ in the Asian populations. Of note, the $T L R+3725 \mathrm{G} / \mathrm{G}$ genotype was beneficially associated with a lower risk of prostate cancer in Sweden [27] and a lower risk of developing chemotherapy-induced neutropenia in children with ALL in Netherland [28], although their etiological relevance to the current findings are unclear. In the present study, the $T L R+3725 \mathrm{G}>\mathrm{C}$ variation did not significantly influence engraftment and relapse rates after SCT.

Bochud, et al.[29] reported that donor TLR4 haplotype S4 among haplotypes S1 to S4 was associated 
Table 5: The results of a multivariate analysis regarding the association between $T L R 1, T L R 2$ and $T L R 4$ variations and clinical outcomes after transplantation in the discovery cohort

\begin{tabular}{|c|c|c|c|c|c|c|c|c|c|c|c|c|}
\hline \multirow[b]{2}{*}{ Variable } & \multicolumn{3}{|c|}{ 5-year OS } & \multicolumn{3}{|c|}{ 5-year PFS } & \multicolumn{3}{|c|}{ 5-year TRM } & \multicolumn{3}{|c|}{ 5-year Relapse } \\
\hline & HR & $95 \% \mathrm{CI}$ & $P$ & HR & $95 \%$ CI & $P$ & HR & $\begin{array}{c}95 \% \\
\text { CI }\end{array}$ & $P$ & HR & $\begin{array}{c}95 \% \\
\text { CI }\end{array}$ & $P$ \\
\hline $\begin{array}{l}\text { Recipient } T L R 1 \\
\text { genotype, G/G } \\
\text { vs. A/A or } \mathrm{A} / \mathrm{G}\end{array}$ & 1.00 & $\begin{array}{c}0.74- \\
1.40\end{array}$ & 0.96 & 1.00 & $\begin{array}{c}0.70- \\
1.40\end{array}$ & 0.97 & 1.29 & $\begin{array}{l}0.80- \\
2.00\end{array}$ & 0.31 & 0.63 & $\begin{array}{c}0.31- \\
1.30\end{array}$ & 0.21 \\
\hline $\begin{array}{l}\text { Donor TLR1 } \\
\text { genotype, } \\
\text { G/G vs. A/A or } \\
\text { A/G }\end{array}$ & 1.09 & $\begin{array}{c}0.80- \\
1.40\end{array}$ & 0.59 & 1.16 & $\begin{array}{c}0.81- \\
1.60\end{array}$ & 0.42 & 1.22 & $\begin{array}{l}0.78- \\
1.92\end{array}$ & 0.38 & 1.06 & $\begin{array}{l}0.55- \\
2.00\end{array}$ & 0.87 \\
\hline $\begin{array}{l}\text { Recipient } T L R 2 \\
\text { genotype, G/G } \\
v s . T / T \text { or } G / T\end{array}$ & 1.02 & $\begin{array}{c}0.59- \\
1.80\end{array}$ & 0.95 & 1.04 & $\begin{array}{c}0.71- \\
1.50\end{array}$ & 0.86 & 1.34 & $\begin{array}{l}0.83- \\
2.10\end{array}$ & 0.23 & 0.61 & $\begin{array}{c}0.24- \\
1.50\end{array}$ & 0.28 \\
\hline $\begin{array}{l}\text { Donor TLR2 } \\
\text { genotype, } \\
\text { G/G vs. T/T or } \\
\text { G/T }\end{array}$ & 1.24 & $\begin{array}{l}0.71- \\
2.10\end{array}$ & 0.45 & 1.24 & $\begin{array}{c}0.85- \\
1.80\end{array}$ & 0.28 & 1.17 & $\begin{array}{c}0.71- \\
1.90\end{array}$ & 0.54 & 0.84 & $\begin{array}{c}0.40- \\
1.80\end{array}$ & 0.65 \\
\hline $\begin{array}{l}\text { Recipient } T L R 4 \\
\text { genotype, G/G } \\
\text { vs. C/C or C/G }\end{array}$ & 1.00 & $\begin{array}{c}0.74- \\
1.30\end{array}$ & 0.98 & 1.06 & $\begin{array}{c}0.75- \\
1.50\end{array}$ & 0.74 & 1.09 & $\begin{array}{c}0.68- \\
1.73\end{array}$ & 0.72 & 0.65 & $\begin{array}{c}0.34- \\
1.30\end{array}$ & 0.20 \\
\hline $\begin{array}{l}\text { Donor TLR4 } \\
\text { genotype, } \\
\text { G/G vs. C/C or } \\
\text { C/G }\end{array}$ & 0.75 & $\begin{array}{c}0.55- \\
1.00\end{array}$ & 0.061 & 0.57 & $\begin{array}{l}0.40- \\
0.82\end{array}$ & 0.0022 & 0.63 & $\begin{array}{c}0.40- \\
1.00\end{array}$ & 0.057 & 0.62 & $\begin{array}{c}0.31- \\
1.20\end{array}$ & 0.17 \\
\hline Recipient age & 1.03 & $\begin{array}{l}1.00- \\
1.00\end{array}$ & $<0.001$ & 1.03 & $\begin{array}{l}1.00- \\
1.00\end{array}$ & $<0.001$ & 1.04 & $\begin{array}{l}1.00- \\
1.10\end{array}$ & $<0.001$ & 1.01 & $\begin{array}{l}0.98- \\
1.05\end{array}$ & 0.38 \\
\hline Year of HSCT & 0.78 & $\begin{array}{l}0.55- \\
1.10\end{array}$ & 0.17 & & & & 0.52 & $\begin{array}{l}0.31- \\
0.89\end{array}$ & 0.016 & & & \\
\hline \multicolumn{13}{|l|}{$\begin{array}{l}\text { Recipient/Donor } \\
\text { sex match }\end{array}$} \\
\hline Female/Male & & & & & & & & & & 0.36 & $\begin{array}{l}0.13- \\
1.00\end{array}$ & 0.053 \\
\hline Male/Female & & & & & & & & & & 0.13 & $\begin{array}{l}0.018- \\
0.95\end{array}$ & 0.044 \\
\hline $\begin{array}{l}\text { Disease stage } \\
\text { Standard risk/ } \\
\text { High risk }\end{array}$ & 2.01 & $\begin{array}{l}1.50- \\
2.70\end{array}$ & $<0.001$ & 1.90 & $\begin{array}{l}1.30- \\
2.70\end{array}$ & $<0.001$ & 2.06 & $\begin{array}{l}1.30- \\
3.30\end{array}$ & 0.0021 & & & \\
\hline $\begin{array}{l}\text { ABO Major } \\
\text { mismatch }\end{array}$ & & & & & & & 0.69 & $\begin{array}{c}0.34- \\
1.40\end{array}$ & 0.29 & & & \\
\hline $\begin{array}{l}\text { ABO Minor } \\
\text { mismatch }\end{array}$ & & & & & & & 1.80 & $\begin{array}{l}0.98- \\
3.30\end{array}$ & 0.058 & & & \\
\hline $\begin{array}{l}\text { ABO } \\
\text { Bidirectional }\end{array}$ & & & & & & & $<0.001$ & $\begin{array}{c}0.00- \\
0.00\end{array}$ & 0.00 & & & \\
\hline $\begin{array}{l}\text { Conditioning } \\
\text { regimen } \\
\text { MAC/RIC }\end{array}$ & & & & & & & 0.64 & $\begin{array}{c}0.30- \\
1.4\end{array}$ & 0.24 & & & \\
\hline
\end{tabular}

(Continued) 


\begin{tabular}{|c|c|c|c|c|c|c|c|c|c|c|c|c|}
\hline \multirow[b]{2}{*}{ Variable } & \multicolumn{3}{|c|}{ 5-year OS } & \multicolumn{3}{|c|}{ 5-year PFS } & \multicolumn{3}{|c|}{ 5-year TRM } & \multicolumn{3}{|c|}{ 5-year Relapse } \\
\hline & HR & $95 \% \mathrm{CI}$ & $P$ & HR & $95 \% \mathrm{CI}$ & $P$ & HR & $\begin{array}{c}95 \% \\
\text { CI }\end{array}$ & $P$ & HR & $\begin{array}{c}95 \% \\
\text { CI }\end{array}$ & $P$ \\
\hline \multicolumn{13}{|c|}{$\begin{array}{l}\text { Pretransplantation } \\
\text { CMV serostatus }\end{array}$} \\
\hline $\begin{array}{l}\text { CMV-positive } \\
\text { recipient }\end{array}$ & & & & & & & & & & 1.70 & $\begin{array}{c}0.54- \\
5.3\end{array}$ & 0.36 \\
\hline Missing & & & & & & & & & & 24.15 & $7.0-84$ & $<0.001$ \\
\hline $\mathrm{TNC}$ & 1.00 & $1.0-1.0$ & 0.38 & 1.00 & $1.0-1.0$ & 0.90 & 1.00 & $1.0-1.0$ & 0.33 & & & \\
\hline
\end{tabular}

TNC, total number of nucleated cells harvested.

Underlined and bold results regarding the genotype represent $P<0.05$.

\begin{tabular}{|c|c|c|c|c|c|c|c|c|c|}
\hline \multirow{2}{*}{ Variable } & \multicolumn{3}{|c|}{ Grades II-IV acute GVHD } & \multicolumn{3}{|c|}{ Grades III-IV acute GVHD } & \multicolumn{3}{|c|}{ Chronic GVHD } \\
\hline & HR & $95 \% \mathrm{CI}$ & $P$ & HR & $95 \% \mathrm{CI}$ & $P$ & HR & $95 \% \mathrm{CI}$ & $P$ \\
\hline $\begin{array}{l}\text { Recipient } T L R 1 \\
\text { genotype, } \\
\text { G/G vs. A/A or } \\
\text { A/G }\end{array}$ & 1.21 & $0.83-1.80$ & 0.33 & 1.57 & $0.87-2.80$ & 0.13 & 1.05 & $0.75-1.50$ & 0.77 \\
\hline $\begin{array}{l}\text { Donor TLR1 } \\
\text { genotype, } \\
\text { G/G vs. A/A or } \\
\text { A/G }\end{array}$ & 0.89 & $0.61-1.30$ & 0.55 & 0.93 & $0.51-1.70$ & 0.80 & 0.76 & $0.53-1.10$ & 0.12 \\
\hline $\begin{array}{l}\text { Recipient } T L R 2 \\
\text { genotype, } \\
\text { G/G vs. T/T or } \\
\text { G/T }\end{array}$ & 0.67 & $0.43-1.10$ & 0.084 & 0.53 & $0.25-1.10$ & 0.097 & 0.82 & $0.56-1.20$ & 0.30 \\
\hline $\begin{array}{l}\text { Donor TLR2 } \\
\text { genotype, } \\
\text { G/G vs. T/T or } \\
\text { G/T }\end{array}$ & 0.62 & $0.41-0.95$ & 0.028 & 0.70 & $0.36-1.40$ & 0.31 & 0.97 & $0.68-1.40$ & 0.87 \\
\hline $\begin{array}{l}\text { Recipient } T L R 4 \\
\text { genotype, } \\
\text { G/G vs. C/C or } \\
\text { C/G }\end{array}$ & 0.86 & $0.59-1.30$ & 0.43 & 1.21 & $0.65-2.20$ & 0.55 & 1.07 & $1.00-2.20$ & 0.043 \\
\hline $\begin{array}{l}\text { Donor TLR4 } \\
\text { genotype, } \\
\text { G/G vs. C/C or } \\
\text { C/G }\end{array}$ & 0.84 & $0.58-1.20$ & 0.35 & 0.67 & $0.37-1.20$ & 0.18 & 0.87 & $0.62-1.20$ & 0.43 \\
\hline Recipient age & 0.99 & $0.98-1.00$ & 0.11 & & & & & & \\
\hline Donor age & 1.03 & $1.00-1.10$ & 0.022 & & & & & & \\
\hline $\begin{array}{l}\text { Disease stage } \\
\text { Standard risk/ } \\
\text { High risk }\end{array}$ & 1.22 & $0.84-1.80$ & 0.31 & 1.54 & $0.85-2.80$ & 0.16 & & & \\
\hline $\begin{array}{l}\text { Myeloid } \\
\text { malignancies }\end{array}$ & & & & & & & 1.50 & $1.00-2.20$ & 0.043 \\
\hline TNC & 1.00 & $1.00-1.00$ & 0.15 & & & & & & \\
\hline
\end{tabular}

TNC, total number of nucleated cells harvested.

Underlined and bold results regarding the genotype represent $P<0.05$. 

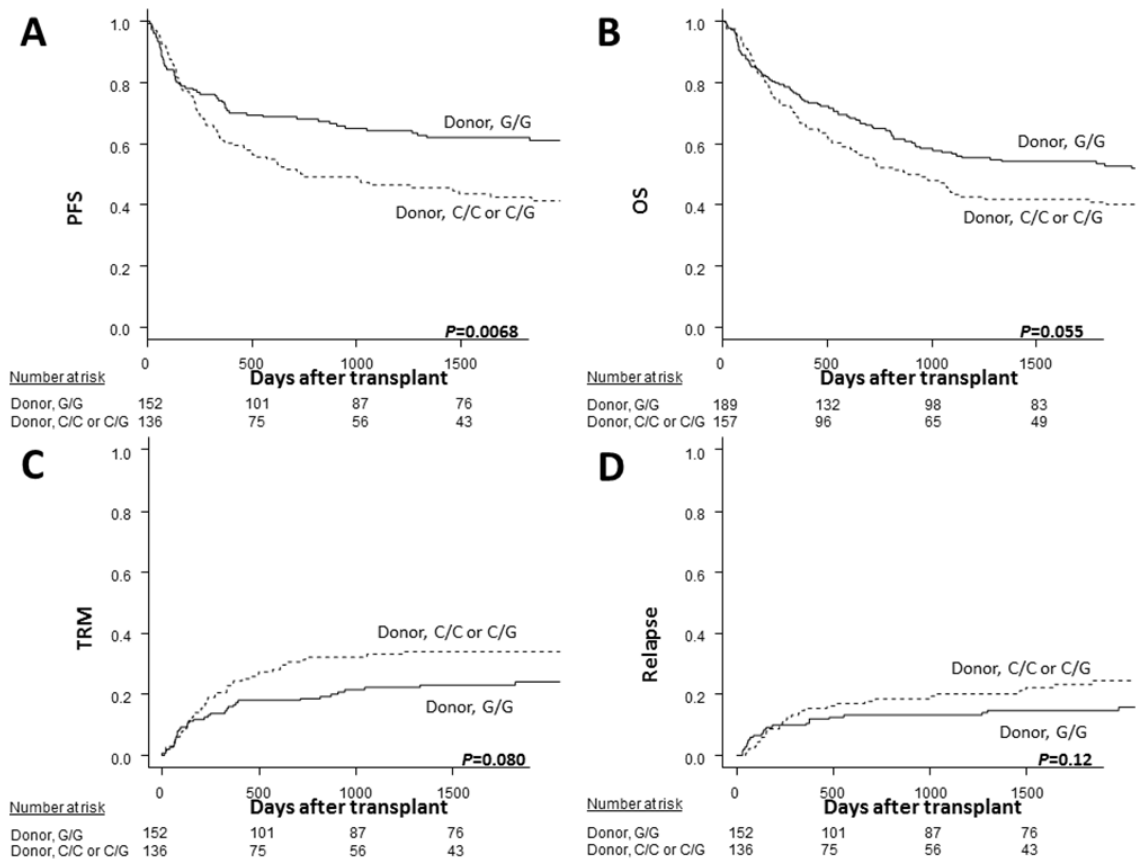

Figure 1: The Kaplan-Meier analysis of the progression-free survival rates (A) and the overall survival rates (B), and the estimated incidence curves of transplant-related mortality (C) and disease relapse (D) after transplantation according to the donor TLR4 genotype in the discovery cohort. The solid lines represent the donor $\mathrm{G} / \mathrm{G}$ genotype, and the dashed lines represent the donor $\mathrm{C} / \mathrm{C}$ or $\mathrm{C} / \mathrm{G}$ genotype.

A

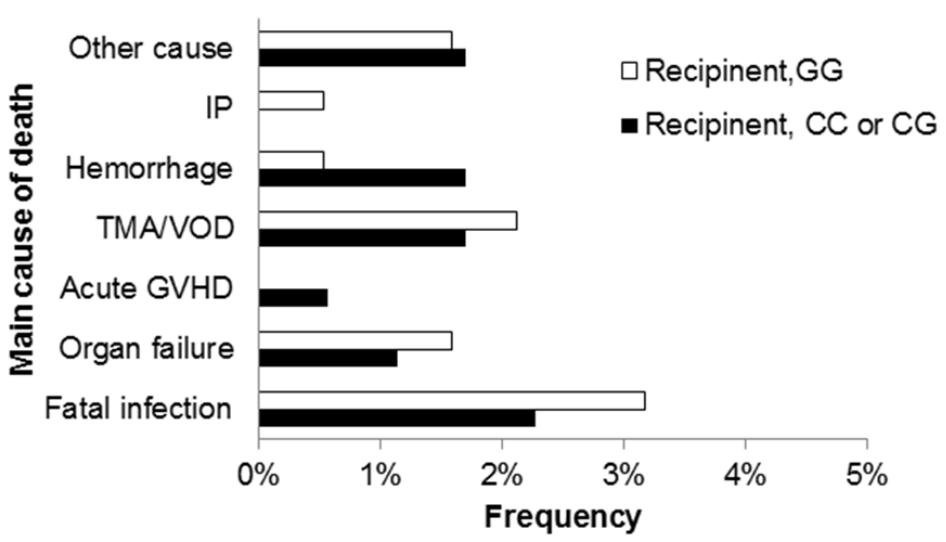

B

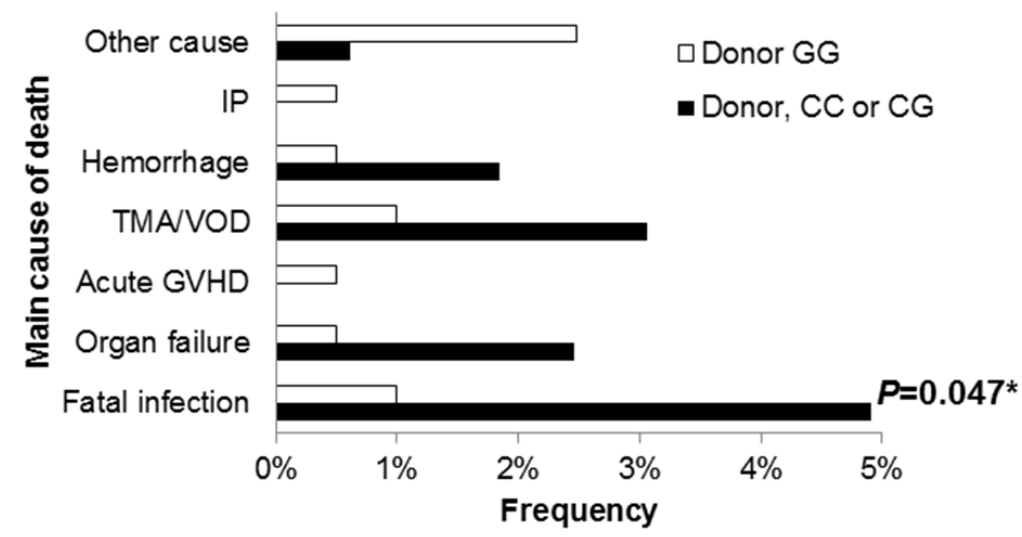

Figure 2: The main causes of death after transplantation according to the recipient (A) and donor TLR 4 genotype (B) in the discovery cohort. The asterisk denotes $P<0.05$. 


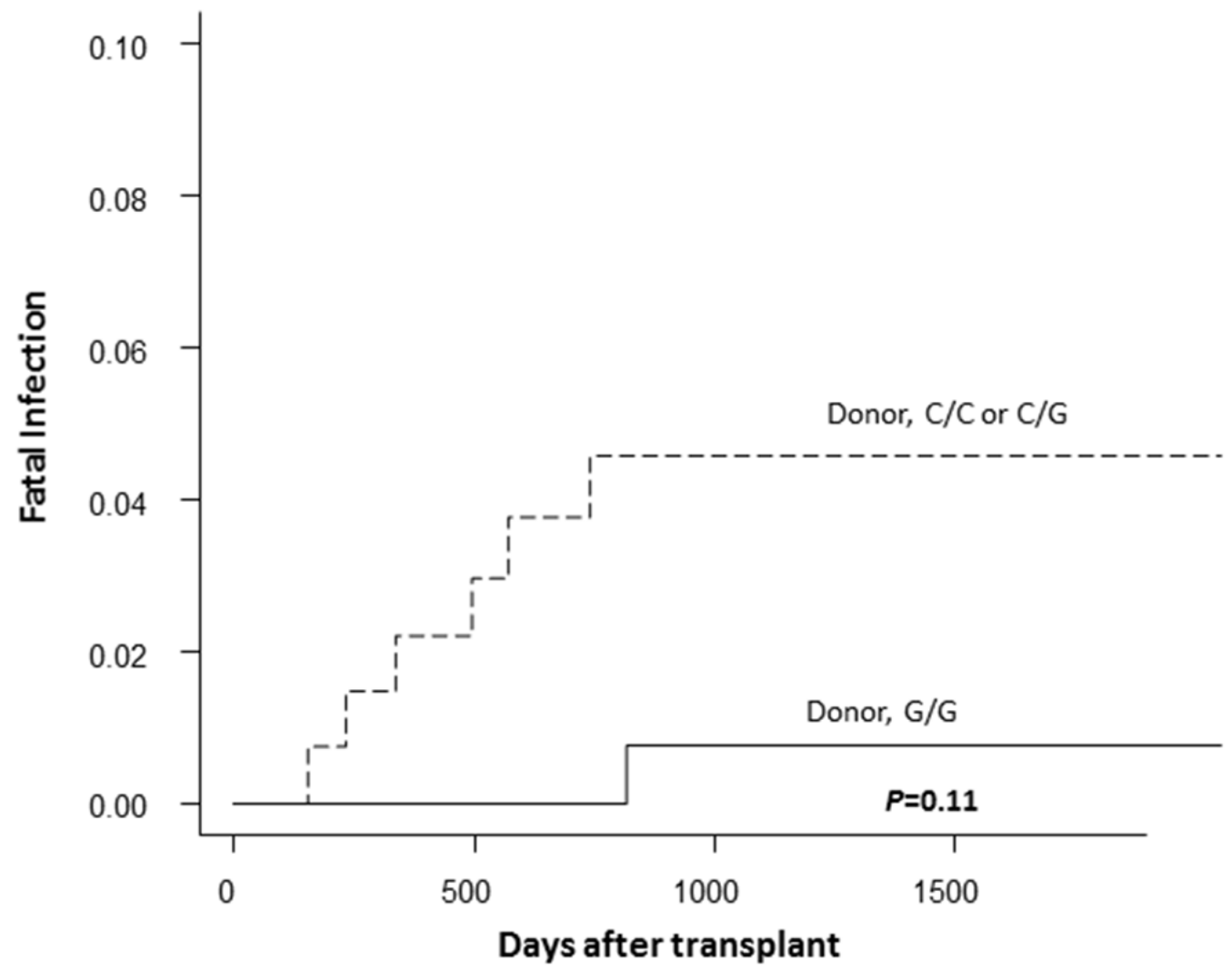

Figure 3: The cumulative incidence of fatal infection after transplantation according to the donor TLR4 genotype in the discovery cohort. The solid lines represent the donor $\mathrm{G} / \mathrm{G}$ genotype, and the dashed lines represent the donor $\mathrm{C} / \mathrm{C}$ or $\mathrm{C} / \mathrm{G}$ genotype.

A

B
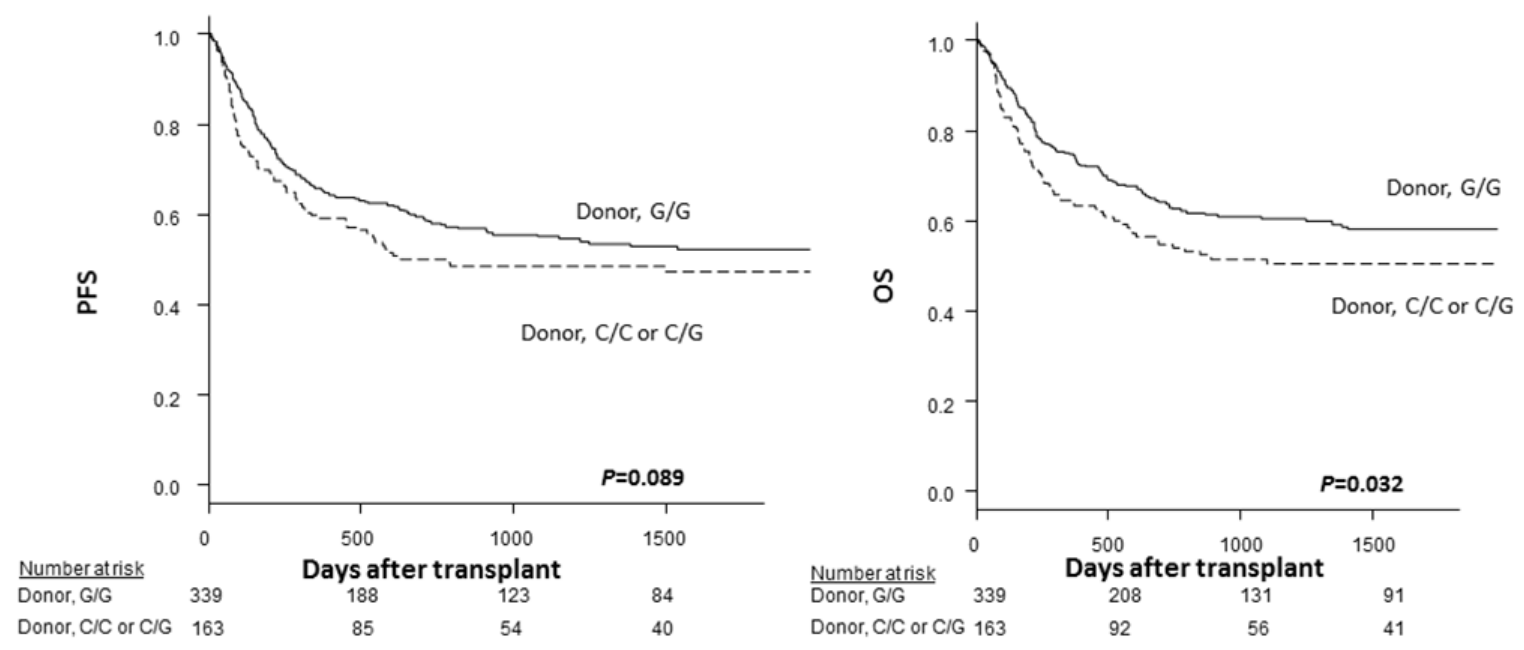

Figure 4: The Kaplan-Meier analysis of the progression-free survival rates (A) and the overall survival rates (B) after transplantation according to the donor TLR4 genotype in the validation cohort. The solid lines represent the donor G/G genotype, and the dashed lines represent the donor $\mathrm{C} / \mathrm{C}$ or $\mathrm{C} / \mathrm{G}$ genotype. 
Table 6: The results of a univariate analysis regarding the association between $T L R 4$ variations and clinical outcomes after transplantation in the validation cohort

\begin{tabular}{|c|c|c|c|c|c|c|c|c|c|}
\hline Variable & n & 5-Year OS, \% & $\boldsymbol{P}$ & 5-Year PFS, \% & $P$ & 5-Year TRM, \% & $P$ & 5-Year Relapse, \% & $\boldsymbol{P}$ \\
\hline \multicolumn{10}{|c|}{ Recipient $T L R 4$ genotype } \\
\hline G/G & 344 & 55 & & 50 & & 28 & & 21 & \\
\hline $\mathrm{C} / \mathrm{G}$ & 143 & 56 & 1.0 & 50 & 1.0 & 29 & 1.0 & 21 & 1.0 \\
\hline $\mathrm{C} / \mathrm{C}$ & 15 & 60 & 1.0 & 60 & 1.0 & 20 & 1.0 & 20 & 1.0 \\
\hline G/G & 344 & 55 & & 50 & & 28 & & 21 & \\
\hline $\mathrm{C} / \mathrm{G}$ or $\mathrm{C} / \mathrm{C}$ & 158 & 56 & 0.94 & 51 & 0.78 & 28 & 0.94 & 21 & 0.90 \\
\hline \multicolumn{10}{|c|}{ Donor TLR4 genotype } \\
\hline G/G & 339 & 58 & & 52 & & 27 & & 21 & \\
\hline $\mathbf{C} / \mathbf{G}$ & 135 & 51 & 0.13 & 48 & 0.39 & 30 & 0.72 & 22 & 1.0 \\
\hline $\mathrm{C} / \mathrm{C}$ & 28 & 50 & 0.54 & 42 & 0.57 & 39 & 0.64 & 20 & 1.0 \\
\hline G/G & 339 & 58 & & 52 & & 27 & & 21 & \\
\hline $\mathrm{C} / \mathrm{G}$ or $\mathrm{C} / \mathrm{C}$ & 163 & 51 & 0.032 & 47 & 0.089 & 31 & 0.22 & 22 & 0.58 \\
\hline
\end{tabular}

Underlined and bold results represent $P<0.05$.

\begin{tabular}{|c|c|c|c|c|c|}
\hline Variable & $\mathbf{n}$ & Grade II-IV aGVHD, \% & $\boldsymbol{P}$ & Chronic GVHD, \% & $P$ \\
\hline \multicolumn{6}{|c|}{ Recipient $T L R 4$ genotype } \\
\hline $\mathbf{G} / \mathbf{G}$ & 344 & 49 & & 42 & \\
\hline $\mathbf{C} / \mathbf{G}$ & 143 & 50 & 1.0 & 38 & 0.84 \\
\hline $\mathrm{C} / \mathrm{C}$ & 15 & 40 & 1.0 & 27 & 0.84 \\
\hline G/G & 344 & 49 & & 42 & \\
\hline $\mathrm{C} / \mathrm{G}$ or $\mathrm{C} / \mathrm{C}$ & 158 & 49 & 0.59 & 37 & 0.38 \\
\hline \multicolumn{6}{|c|}{ Donor $T L R 4$ genotype } \\
\hline G/G & 339 & 49 & & 38 & \\
\hline $\mathbf{C} / \mathbf{G}$ & 135 & 49 & 1.0 & 50 & 0.13 \\
\hline $\mathrm{C} / \mathrm{C}$ & 28 & 50 & 1.0 & 25 & 0.13 \\
\hline $\mathbf{G} / \mathbf{G}$ & 339 & 49 & & 38 & \\
\hline $\mathrm{C} / \mathrm{G}$ or $\mathrm{C} / \mathrm{C}$ & 163 & 49 & 0.95 & 45 & 0.31 \\
\hline
\end{tabular}

with a risk of invasive aspergillosis after allogeneic hematopoietic stem cell transplantation. However, because haplotype $\mathrm{S} 4$ is absent in Asian populations [30, 31], which is defined by the TLR4 variations rs4986790 [D299G] and rs4986791 [T399I], but not by TLR4 variation rs11536889 that was investigated in the present study, we decided not to investigate the potential link between each haplotype and the transplant outcomes.

One major limitation associated with the present study is that the detailed information on the infections, including the types, severity, treatments, and therapeutic appropriateness, was beyond the scope of the current study and the only the information on the causes of death (such as fatal infections) was available.

In conclusion, the findings of the present data suggested that the donor TLR $4+3725 \mathrm{G}>\mathrm{C}$ variations predicted better survival outcomes after SCT than other genotypes. Therefore, TLR $4+3725 \mathrm{G}>\mathrm{C}$ genotyping in donors may be a valuable tool for selecting donors and evaluating pretransplantation risks that, combined with other currently known risk factors, can form the basis for carrying out suitable tailoring of transplantation strategies. Considering the plausible functional roles of these variations, they may be candidates for future 
Table 7: The results of a multivariate analysis regarding the association between $T L R 4$ variations and the clinical outcomes after transplantation in the validation cohort

\begin{tabular}{|c|c|c|c|c|c|c|c|c|c|c|c|c|}
\hline \multirow[b]{2}{*}{ Variable } & \multicolumn{3}{|c|}{ 5-year OS } & \multicolumn{3}{|c|}{ 5-year PFS } & \multicolumn{3}{|c|}{ 5-year TRM } & \multicolumn{3}{|c|}{ 5-year Relapse } \\
\hline & $\begin{array}{c}\text { Adjusted } \\
\text { HR }\end{array}$ & $95 \% \mathrm{CI}$ & $P$ & $\begin{array}{c}\text { Adjusted } \\
\text { HR }\end{array}$ & $95 \% \mathrm{CI}$ & $P$ & $\begin{array}{c}\text { Adjusted } \\
\text { HR }\end{array}$ & $95 \% \mathrm{CI}$ & $P$ & $\begin{array}{c}\text { Adjusted } \\
\text { HR }\end{array}$ & $95 \% \mathrm{CI}$ & $\boldsymbol{P}$ \\
\hline $\begin{array}{l}\text { Recipient } \\
\text { TLR4 } \\
\text { genotype, } \\
\text { G/G vs. C/C } \\
\text { or C/G }\end{array}$ & 1.0 & $\begin{array}{c}0.75- \\
1.3\end{array}$ & 1.0 & 0.97 & $0.74-1.3$ & 0.83 & 0.98 & $\begin{array}{c}0.68- \\
1.4\end{array}$ & 0.90 & 0.98 & $\begin{array}{c}0.64- \\
1.5\end{array}$ & 0.93 \\
\hline $\begin{array}{l}\text { Donor } \\
\text { TLR4 } \\
\text { genotype, } \\
\text { G/G vs. C/C } \\
\text { or C/G }\end{array}$ & 0.75 & $\begin{array}{c}0.56- \\
0.99\end{array}$ & 0.043 & 0.81 & $0.62-1.1$ & 0.12 & 0.81 & $\begin{array}{c}0.57- \\
1.1\end{array}$ & 0.24 & 0.87 & $\begin{array}{c}0.56- \\
1.3\end{array}$ & 0.53 \\
\hline $\begin{array}{l}\text { Recipient } \\
\text { age }\end{array}$ & 1.4 & $1.0-1.8$ & 0.026 & 1.3 & $1.0-1.7$ & 0.033 & 1.9 & $1.3-2.7$ & 0.00038 & 0.75 & $\begin{array}{c}0.50- \\
1.1\end{array}$ & 0.17 \\
\hline $\begin{array}{l}\text { Recipient/ } \\
\text { Donor sex } \\
\text { match }\end{array}$ & 1.1 & $\begin{array}{c}0.91- \\
1.3\end{array}$ & 0.38 & 1.1 & $0.92-1.3$ & 0.3 & & & & & & \\
\hline $\begin{array}{l}\text { Female/ } \\
\text { Male }\end{array}$ & & & & & & & 0.85 & $\begin{array}{c}0.53- \\
1.4\end{array}$ & 0.49 & 1.1 & $\begin{array}{c}0.67- \\
1.9\end{array}$ & 0.61 \\
\hline $\begin{array}{l}\text { Male/ } \\
\text { Female }\end{array}$ & & & & & & & 1.1 & $\begin{array}{c}0.72- \\
1.8\end{array}$ & 0.57 & 1.3 & $\begin{array}{c}0.75- \\
2.1\end{array}$ & 0.39 \\
\hline
\end{tabular}

Underlined and bold results regarding the genotype represent $P<0.05$.

\begin{tabular}{|c|c|c|c|c|c|c|c|c|c|}
\hline \multirow{2}{*}{ Variable } & \multicolumn{3}{|c|}{ Grades II-IV acute GVHD } & \multicolumn{3}{|c|}{ Grades III-IV acute GVHD } & \multicolumn{3}{|c|}{ Chronic GVHD } \\
\hline & Adjusted HR & $95 \% \mathrm{CI}$ & $\boldsymbol{P}$ & Adjusted HR & $95 \% \mathrm{CI}$ & $P$ & Adjusted HR & $95 \% \mathrm{CI}$ & $\boldsymbol{P}$ \\
\hline $\begin{array}{l}\text { Recipient } T L R 4 \\
\text { genotype, } \mathrm{G} / \mathrm{G} v s \text {. } \\
\mathrm{C} / \mathrm{C} \text { or } \mathrm{C} / \mathrm{G}\end{array}$ & 0.93 & $0.70-1.2$ & 0.61 & 0.94 & $\begin{array}{c}0.58- \\
1.5\end{array}$ & 0.79 & 1.2 & $\begin{array}{c}0.84- \\
1.6\end{array}$ & 0.35 \\
\hline $\begin{array}{l}\text { Donor TLR4 } \\
\text { genotype, } \mathrm{G} / \mathrm{G} v s \text {. } \\
\mathrm{C} / \mathrm{C} \text { or } \mathrm{C} / \mathrm{G}\end{array}$ & 1.0 & $0.78-1.3$ & 0.89 & 0.78 & $\begin{array}{c}0.49- \\
1.2\end{array}$ & 0.29 & 0.85 & $\begin{array}{c}0.62- \\
1.2\end{array}$ & 0.29 \\
\hline Recipient age & 1.0 & $0.82-1.4$ & 0.64 & 1.1 & $\begin{array}{c}0.68- \\
1.7\end{array}$ & 0.76 & 1.2 & $\begin{array}{c}0.92- \\
1.7\end{array}$ & 0.15 \\
\hline \multicolumn{10}{|l|}{$\begin{array}{l}\text { Recipient/Donor } \\
\text { sex match }\end{array}$} \\
\hline Female/Male & 1.1 & $0.82-1.6$ & 0.46 & 1.1 & $\begin{array}{c}0.62- \\
2.1\end{array}$ & 0.69 & 1.1 & $\begin{array}{c}0.73- \\
1.6\end{array}$ & 0.70 \\
\hline Male/Female & 0.9 & $0.63-1.3$ & 0.57 & 1.1 & $\begin{array}{c}0.59- \\
2.0\end{array}$ & 0.81 & 0.96 & $\begin{array}{c}0.62- \\
1.5\end{array}$ & 0.85 \\
\hline
\end{tabular}


prophylactic and therapeutic strategies for complications after allogeneic SCT. Further studies are warranted to ascertain whether or not the findings of this study can be extended to other stem cell sources or to HLA-mismatched transplantation and to validate the present findings in other ethnic groups.

\section{PATIENTS AND METHODS}

\section{Patients}

The patients underwent BMT through the JMDP with T cell-replete marrow between May 1993 and November 2005 (Table 1). No patients had a history of any prior transplantation. The final clinical data analyses of these patients were completed by November 25, 2009. The conditioning regimen varied according to the underlying disease and the condition of the patient. The combination of cyclophosphamide (CY) combined with total body irradiation (TBI) was mainly used for the myeloablative conditioning (MAC) regimen, whereas the combination of fludarabine and melphalan or busulfan was mainly used for the reduced-intensity conditioning (RIC) regimen [32]. Cyclosporine or tacrolimus with short-term methotrexate was used for GVHD prophylaxis [33, 34]. No patients received anti-T cell therapy, such as antithymocyte globulin or ex vivo $\mathrm{T}$ cell depletion, in this study. All patients and donors gave their informed consent at the time of transplantation to take part in molecular studies of this nature, in accordance with the Declaration of Helsinki. This project was approved by the Institutional Review Board of Aichi Medical University School of Medicine and the JMDP. All methods were performed in accordance with the approved guidelines and regulations.

For the discovery study, TLR1, TLR2 and $T L R 4$ genotyping was performed in 365 patients with hematologic malignancies and their unrelated and HLA-A, HLA-B, HLA-C, HLA-DRB1, HLA-DQB1 and HLA-DPB1 allele-matched donors. The diagnoses included acute myeloid leukemia (AML) $(n=125$; $34 \%)$, acute lymphoblastic leukemia (ALL) $(n=82$; $22 \%)$, myelodysplastic syndrome (MDS) $(n=57 ; 16 \%)$, malignant lymphoma (ML) $(n=36 ; 9.9 \%)$ and chronic myeloid leukemia (CML) $(n=65 ; 18 \%)$. "Standard risk" included acute leukemia in the first remission, chronic myeloid leukemia in the chronic phase and myelodysplastic syndrome and malignant lymphoma in complete remission. "High risk" included all others. Lymphoid malignancies included ALL and ML.

For the validation study, the TRL4 rs11536889 variations were imputed using the data from the 1000 Genomes Project 36, as described in our previous study [35]. The cohort included 502 patients with hematological malignancies and their unrelated donors who were HLA-A, HLA-B, HLA-C, HLA-DRB1, and HLA-DQB1 allele-matched but who were mismatched by one or two
HLA-DPB1 alleles. The diagnoses included AML ( $\mathrm{n}=149$; $30 \%)$, ALL ( $\mathrm{n}=152 ; 30 \%)$, MDS ( $\mathrm{n}=58 ; 12 \%)$, ML $(\mathrm{n}=61$; $12 \%)$ and CML $(\mathrm{n}=82 ; 16 \%)$.

HLA 12/12-allele-matched transplants were incorporated into the discovery cohort to eliminate the impact of HLA-allele mismatch on transplant outcomes, and all 365 available pairs were analyzed in the discovery cohort study. In conducting the validation study, in order to minimize the influence of HLA on the transplant outcomes, all 502 transplants from HLADPB1-mismatched donors were incorporated into the validation cohort.

\section{Genotyping}

Real-time polymerase chain reaction (PCR) genotyping for TLR1, TLR2 and TLR4 was performed using the TaqMan-Allelic discrimination method in the StepOnePlus Real-Time PCR system (Applied Biosystems, Foster City, CA, USA) as described previously [6], and the results were analyzed using the Allelic Discrimination software program (Applied Biosystems). The specific probe designed for SNP rs5743551 (-7202G>A) (product No. C_1180670_30), rs7656411 (22215G>T) (product No. C__29420880_10), rs11536889 (+3725G $>$ C) (product No. C_ $\left.31784034 \_10\right)$ and TaqMan genotyping master mix were purchased from Applied Biosystems.

\section{Data management and statistical analyses}

Data were collected by the JMDP using a standardized report form. Follow-up reports were submitted at 100 days, 1 year and then annually after transplantation. Only recipients were routinely measured for the pretransplantation cytomegalovirus (CMV) serostatus. The time to neutrophil engraftment was defined as the first of 3 consecutive days with an absolute neutrophil count of more than $0.5 \times 10^{9} / \mathrm{L}$. Acute GVHD developing within the first 100 days posttransplantation was diagnosed and graded based on the established criteria [36]. A classification of chronic GVHD observed in patients who survived beyond day 100 was based on the Seattle criteria [37]. The overall survival (OS) was calculated from the date of transplantation to the date of death from any cause. Disease relapse was defined as the number of days from transplantation to disease relapse or progression. The transplant-related mortality (TRM) was defined as death due to any cause other than relapse or disease progression. The PFS was defined as survival without disease relapse or progression. Any patients who were alive at the last follow-up date were censored. Data regarding the clinical and microbiological characteristics of infections, postmortem changes, prophylaxis against infections and therapy for GVHD given on an institutional basis were not considered in this study. 
All of the statistical analyses were carried out using the EZR software package [38]. The probabilities of OS and PFS were calculated using the Kaplan-Meier method, and comparisons between groups were performed via the log-rank test. The occurrence of TRM, disease relapse, acute GVHD and chronic GVHD were compared using the Gray test [39] and analyzed using the cumulative incidence analysis [40], considering relapse, death without disease relapse, death without acute GVHD, death without chronic GVHD and death without engraftment as respective competing risks. The cumulative incidence of fatal infection was analyzed using the Gray test, while considering fatal infections without disease relapse and relapse or death without fatal infection as competing risks. A multivariate Cox model was constructed for the OS and PFS, and a Fine-Gray competing risk regression model was constructed for TRM, relapse, grades 2-4 acute GVHD, grades 3-4 acute GVHD and chronic GVHD using stepwise selection at a significance level of 5\% to evaluate the hazard ratio (HR) associated with the TLR1, TLR2 and TLR4 genotypes. Recipient age at the time of BMT, sex, pretransplantation CMV serostatus, disease characteristics (i.e. disease type, disease lineage and disease risk at transplantation), donor characteristics (i.e. age, sex compatibility and ABO compatibility), transplant characteristics (i.e. MAC or RIC and total number of nucleated cells harvested per recipient weight) and year of transplantation were used as covariates. Although these covariates were all available for the discovery study, some of these covariates were not available for the validation study (Table 1). The median was used as the cut-off point for continuous variables. The chi-squared and MannWhitney tests were used to compare the results of two groups. The linkage disequilibrium (LD) structure among the single nucleotide polymorphisms in the TLR1, TLR2 and TLR4 gene was determined using HAPLOVIEW, version 4.2 [41, 42]. For all analyses, $P<0.05$ was considered statistically significant.

\section{Author contributions}

A.T and K.U designed the study. A.S-O and Y.N performed the experimental analyses. K.U performed the statistical analysis. K.U and A.T wrote the paper. S.M, M.M, T.H, I.H, J.L. E, M. O, K.K, Y.M, T.F, Y.K, N.D, K.M, T.M, and S.O contributed to data collection and sample management. All authors reviewed the manuscript.

\section{ACKNOWLEDGMENTS AND FUNDING}

We would like to thank Dr Keitaro Matsuo for technical assistance with performing the experiments. We thank the Japan Marrow Donor Program and all of the Japan Marrow Donor Program transplant teams who provided valuable assistance in caring for the patients and donors investigated in this study.
This study was supported by grants from the Ministry of Education, Culture, Sports and Technology of Japan (15K09513), the Ministry of Health, Labour and Welfare of Japan (15eK0510002h0002, $15 \mathrm{ek} 0109134 \mathrm{~h} 0001$, and $16 \mathrm{mk} 0101065 \mathrm{~h} 0001)$, the SENSHIN Medical Research Foundation (Osaka, Japan), the Aichi Cancer Research Foundation (Nagoya, Japan) and the 24th General Assembly of the Japanese Association of Medical Sciences (Nagoya, Japan). The funders played no role in the study design, data collection and analysis, the decision to publish or the preparation of the manuscript.

\section{CONFLICTS OF INTEREST}

The authors declare no competing financial interests.

\section{REFERENCES}

1. Gratwohl A, Brand R, Frassoni F, Rocha V, Niederwieser D, Reusser P, Einsele H, Cordonnier C, and Acute and Chronic Leukemia Working Parties, and Infectious Diseases Working Party of the European Group for Blood and Marrow Transplantation. Cause of death after allogeneic haematopoietic stem cell transplantation (HSCT) in early leukaemias: an EBMT analysis of lethal infectious complications and changes over calendar time. Bone Marrow Transplant. 2005; 36:757-69.

2. Tie R, Zhang T, Yang B, Fu H, Han B, Yu J, Tan Y, Huang H. Clinical implications of HLA locus mismatching in unrelated donor hematopoietic cell transplantation: a metaanalysis. Oncotarget. 2017; 8:27645-60. doi: 10.18632/ oncotarget.15291.

3. Takami A, Espinoza JL, Onizuka M, Ishiyama K, Kawase T, Kanda Y, Sao H, Akiyama H, Miyamura K, Okamoto S, Inoue M, Ohtake S, Fukuda T, et al, and Japan Marrow Donor Program. A single-nucleotide polymorphism of the $\mathrm{Fc} \gamma$ receptor type IIIA gene in the recipient predicts transplant outcomes after HLA fully matched unrelated BMT for myeloid malignancies. Bone Marrow Transplant. 2011; 46:238-43.

4. Nomoto H, Takami A, Espinoza JL, Matsuo K, Mizuno S, Onizuka M, Kashiwase K, Morishima Y, Fukuda T, Kodera Y, Doki N, Miyamura K, Mori T, et al. A donor thrombomodulin gene variation predicts graft-versus-host disease development and mortality after bone marrow transplantation. Int J Hematol. 2015; 102:460-70.

5. Nakata K, Takami A, Espinoza JL, Matsuo K, Morishima Y, Onizuka M, Fukuda T, Kodera Y, Akiyama H, Miyamura K, Mori T, Nakao S, and Japan Marrow Donor Program. The recipient CXCL10 $+1642 \mathrm{C}>\mathrm{G}$ variation predicts survival outcomes after HLA fully matched unrelated bone marrow transplantation. Clin Immunol. 2013; 146:104-11.

6. Espinoza JL, Takami A, Onizuka M, Sao H, Akiyama H, Miyamura K, Okamoto S, Inoue M, Kanda Y, Ohtake S, 
Fukuda T, Morishima Y, Kodera Y, Nakao S, and Japan Marrow Donor Program. NKG2D gene polymorphism has a significant impact on transplant outcomes after HLAfully-matched unrelated bone marrow transplantation for standard risk hematologic malignancies. Haematologica. 2009; 94:1427-34.

7. Espinoza JL, Takami A, Nakata K, Onizuka M, Kawase T, Akiyama H, Miyamura K, Morishima Y, Fukuda T, Kodera Y, Nakao S, and Japan Marrow Donor Program. A genetic variant in the IL-17 promoter is functionally associated with acute graft-versus-host disease after unrelated bone marrow transplantation. PLoS One. 2011; 6:e26229.

8. Rocha V, Franco RF, Porcher R, Bittencourt H, Silva WA Jr, Latouche A, Devergie A, Esperou H, Ribaud P, Socie G, Zago MA, Gluckman E. Host defense and inflammatory gene polymorphisms are associated with outcomes after HLA-identical sibling bone marrow transplantation. Blood. 2002; 100:3908-18.

9. Takeuchi O, Akira S. Pattern recognition receptors and inflammation. Cell. 2010; 140:805-20.

10. Vo MC, Lee HJ, Kim JS, Hoang MD, Choi NR, Rhee JH, Lakshmanan VK, Shin SJ, Lee JJ. Dendritic cell vaccination with a toll-like receptor agonist derived from mycobacteria enhances anti-tumor immunity. Oncotarget. 2015; 6:3378190. doi: 10.18632/oncotarget.5281.

11. Heidegger $S$, van den Brink MR, Haas T, Poeck H. The role of pattern-recognition receptors in graft-versus-host disease and graft-versus-leukemia after allogeneic stem cell transplantation. Front Immunol. 2014; 5:337.

12. Maeda Y. Pathogenesis of graft-versus-host disease: innate immunity amplifying acute alloimmune responses. Int $\mathrm{J}$ Hematol. 2013; 98:293-99.

13. Penack O, Holler E, van den Brink MR. Graft-versus-host disease: regulation by microbe-associated molecules and innate immune receptors. Blood. 2010; 115:1865-72.

14. Wurfel MM, Gordon AC, Holden TD, Radella F, Strout J, Kajikawa O, Ruzinski JT, Rona G, Black RA, Stratton S, Jarvik GP, Hajjar AM, Nickerson DA, et al. Toll-like receptor 1 polymorphisms affect innate immune responses and outcomes in sepsis. Am J Respir Crit Care Med. 2008; 178:710-20.

15. Tahara T, Shibata T, Wang F, Yamashita H, Hirata I, Arisawa T. Genetic Polymorphisms of Molecules Associated with Innate Immune Responses, TRL2 and MBL2 Genes in Japanese Subjects with Functional Dyspepsia. J Clin Biochem Nutr. 2010; 47:217-23.

16. Sato K, Yoshimura A, Kaneko T, Ukai T, Ozaki Y, Nakamura H, Li X, Matsumura H, Hara Y, Ogata Y. A single nucleotide polymorphism in 3'-untranslated region contributes to the regulation of Toll-like receptor 4 translation. J Biol Chem. 2012; 287:25163-72.

17. Spiller S, Elson G, Ferstl R, Dreher S, Mueller T, Freudenberg M, Daubeuf B, Wagner H, Kirschning CJ.
TLR4-induced IFN-gamma production increases TLR2 sensitivity and drives Gram-negative sepsis in mice. J Exp Med. 2008; 205:1747-54.

18. Daubeuf B, Mathison J, Spiller S, Hugues S, Herren S, Ferlin W, Kosco-Vilbois M, Wagner H, Kirschning CJ, Ulevitch R, Elson G. TLR4/MD-2 monoclonal antibody therapy affords protection in experimental models of septic shock. J Immunol. 2007; 179:6107-14.

19. Lima CX, Souza DG, Amaral FA, Fagundes CT, Rodrigues IP, Alves-Filho JC, Kosco-Vilbois M, Ferlin W, Shang L, Elson G, Teixeira MM. Therapeutic Effects of Treatment with Anti-TLR2 and Anti-TLR4 Monoclonal Antibodies in Polymicrobial Sepsis. PLoS One. 2015; 10:e0132336.

20. Wang H, Wei Y, Zeng Y, Qin Y, Xiong B, Qin G, Li J, Hu D, Qiu X, Sooranna SR, Pinhu L. The association of polymorphisms of TLR4 and CD14 genes with susceptibility to sepsis in a Chinese population. BMC Med Genet. 2014; 15:123.

21. Mansur A, von Gruben L, Popov AF, Steinau M, Bergmann I, Ross D, Ghadimi M, Beissbarth T, Bauer M, Hinz J. The regulatory toll-like receptor 4 genetic polymorphism rs11536889 is associated with renal, coagulation and hepatic organ failure in sepsis patients. J Transl Med. 2014; 12:177.

22. Hishida A, Matsuo K, Goto Y, Mitsuda Y, Hiraki A, Naito M, Wakai K, Tajima K, Hamajima N. Toll-like receptor $4+3725 \mathrm{G} / \mathrm{C}$ polymorphism, Helicobacter pylori seropositivity, and the risk of gastric atrophy and gastric cancer in Japanese. Helicobacter. 2009; 14:47-53.

23. Hishida A, Matsuo K, Goto Y, Naito M, Wakai K, Tajima K, Hamajima N. Combined effect of miR-146a rs2910164 G/C polymorphism and Toll-like receptor $4+3725$ G/C polymorphism on the risk of severe gastric atrophy in Japanese. Dig Dis Sci. 2011; 56:1131-37.

24. Zhou L, Wei B, Xing C, Xie H, Yu X, Wu L, Zheng S. Polymorphism in 3'-untranslated region of toll-like receptor 4 gene is associated with protection from hepatitis B virus recurrence after liver transplantation. Transpl Infect Dis. $2011 ; 13: 250-58$.

25. Fukusaki T, Ohara N, Hara Y, Yoshimura A, Yoshiura K. Evidence for association between a Toll-like receptor 4 gene polymorphism and moderate/severe periodontitis in the Japanese population. J Periodontal Res. 2007; 42:541-45.

26. Ding YS, Zhao Y, Xiao YY, Zhao G. Toll-like receptor 4 gene polymorphism is associated with chronic periodontitis. Int J Clin Exp Med. 2015; 8:6186-92.

27. Zheng SL, Augustsson-Bälter K, Chang B, Hedelin M, Li L, Adami HO, Bensen J, Li G, Johnasson JE, Turner AR, Adams TS, Meyers DA, Isaacs WB, et al. Sequence variants of toll-like receptor 4 are associated with prostate cancer risk: results from the CAncer Prostate in Sweden Study. Cancer Res. 2004; 64:2918-22. 
28. Miedema KG, te Poele EM, Tissing WJ, Postma DS, Koppelman GH, de Pagter AP, Kamps WA, Alizadeh BZ, Boezen HM, de Bont ES. Association of polymorphisms in the TLR4 gene with the risk of developing neutropenia in children with leukemia. Leukemia. 2011; 25:995-1000.

29. Bochud PY, Chien JW, Marr KA, Leisenring WM, Upton A, Janer M, Rodrigues SD, Li S, Hansen JA, Zhao LP, Aderem A, Boeckh M. Toll-like receptor 4 polymorphisms and aspergillosis in stem-cell transplantation. N Engl J Med. 2008; 359:1766-77.

30. Asakura Y, Komatsu T. Toll-like receptor 4 polymorphisms and aspergillosis. N Engl J Med. 2009; 360:635.

31. Aki K, Okubo Y, Nanjo H, Ishiwatari T, Nihonyanagi Y, Tochigi N, Wakayama M, Nemoto T, Fukutake K, Shinozaki M, Hori Y, Masuda H, Shibuya K. Genomic Analysis of Single Nucleotide Polymorphisms Asp299Gly and Thr399Ile in Japanese Patients with Invasive Aspergillosis. Jpn J Infect Dis. 2015; 68:330-32.

32. Giralt S, Ballen K, Rizzo D, Bacigalupo A, Horowitz M, Pasquini M, Sandmaier B. Reduced-intensity conditioning regimen workshop: defining the dose spectrum. Report of a workshop convened by the center for international blood and marrow transplant research. Biol Blood Marrow Transplant. 2009; 15:367-69.

33. Storb R, Deeg HJ, Whitehead J, Appelbaum F, Beatty P, Bensinger W, Buckner CD, Clift R, Doney K, Farewell V, Hansen J, Hill R, Lum L, et al. Methotrexate and cyclosporine compared with cyclosporine alone for prophylaxis of acute graft versus host disease after marrow transplantation for leukemia. N Engl J Med. 1986; 314:729-35.

34. Nash RA, Antin JH, Karanes C, Fay JW, Avalos BR, Yeager AM, Przepiorka D, Davies S, Petersen FB, Bartels P, Buell D, Fitzsimmons W, Anasetti C, et al. Phase 3 study comparing methotrexate and tacrolimus with methotrexate and cyclosporine for prophylaxis of acute graft-versus-host disease after marrow transplantation from unrelated donors. Blood. 2000; 96:2062-68.

35. Sato-Otsubo A, Nannya Y, Kashiwase K, Onizuka M, Azuma F, Akatsuka Y, Ogino Y, Satake M, Sanada M, Chiba S, Saji H, Inoko H, Kennedy GC, et al, and Japan Marrow Donor Program. Genome-wide surveillance of mismatched alleles for graft-versus-host disease in stem cell transplantation. Blood. 2015; 126:2752-63.

36. Przepiorka D, Weisdorf D, Martin P, Klingemann HG, Beatty P, Hows J, Thomas ED. 1994 Consensus Conference on Acute GVHD Grading. Bone Marrow Transplant. 1995; 15:825-28.

37. Shulman HM, Sullivan KM, Weiden PL, McDonald GB, Striker GE, Sale GE, Hackman R, Tsoi MS, Storb R, Thomas ED. Chronic graft-versus-host syndrome in man. A long-term clinicopathologic study of 20 Seattle patients. Am J Med. 1980; 69:204-17.

38. Kanda Y. Investigation of the freely available easy-touse software 'EZR' for medical statistics. Bone Marrow Transplant. 2013; 48:452-58.

39. Gooley TA, Leisenring W, Crowley J, Storer BE. Estimation of failure probabilities in the presence of competing risks: new representations of old estimators. Stat Med. 1999; 18:695-706.

40. Scrucca L, Santucci A, Aversa F. Competing risk analysis using R: an easy guide for clinicians. Bone Marrow Transplant. 2007; 40:381-87.

41. Barrett JC, Fry B, Maller J, Daly MJ. Haploview: analysis and visualization of LD and haplotype maps. Bioinformatics. 2005; 21:263-65.

42. Stephens M, Scheet P. Accounting for decay of linkage disequilibrium in haplotype inference and missing-data imputation. Am J Hum Genet. 2005; 76:449-62. 